\title{
Medo de Falar em Público em uma Amostra da População: Prevalência, Impacto no Funcionamento Pessoal e Tratamento
}

\author{
Gustavo J. Fonseca D’El Rey ${ }^{1}$ \\ Carla Alessandra Pacini \\ Centro de Pesquisas e Tratamento de Transtornos de Ansiedade - SP
}

\begin{abstract}
RESUMO - O medo de falar em público constitui um subtipo não reconhecido da fobia social em estudos epidemiológicos. Para se verificar a prevalência, o impacto no funcionamento pessoal e a procura por tratamento do medo de falar em público, foi realizada uma pesquisa com 452 residentes da cidade de São Paulo, Brasil. Trinta e dois porcento dos entrevistados reportaram ansiedade excessiva quando falavam para um grande grupo de pessoas. No total, $13 \%$ dos entrevistados relataram que o medo de falar em público resultou em grande interferência em seu trabalho, vida social e educação, ou causou sofrimento acentuado. Esta pesquisa apóia a inclusão de formas graves do medo de falar em público no constructo diagnóstico da fobia social e sugere, também, que essa ansiedade de falar em público pode ter um impacto negativo na vida de muitos indivíduos na comunidade.
\end{abstract}

Palavras-chave: medo de falar em público; fobia social; prevalência; impacto no funcionamento.

\section{Public-Speaking Fear in a Population Sample: Prevalence, Impact on Personnel Functioning and Treatment}

\begin{abstract}
The public-speaking fear constitutes a recognizable subtype of social phobia in epidemiologic studies. To verify the impact on personnel functioning and the professional help seeking for treatment of public-speaking fear in a population sample, we conducted a survey with 452 residents of the city of São Paulo, Brazil. Thirty two per cent of the respondents reported that had excessive anxiety when spoke to a large audience. In total, $13 \%$ of the survey reported that public-speaking fear had resulted in a marked interference in their work, social life and education, or had caused then marked distress. Seven per cent of the survey had public-speaking fear in isolation, without evidence of others social fears. This data support the inclusion of severe forms of public-speaking fear within the social phobia diagnostic construct and also suggest that publicspeaking anxiety may have a negative impact on the lives of many individuals in the community.
\end{abstract}

Key words: public-speaking fear; social phobia; prevalence; impact on functioning.

A fobia social é muitas vezes confundida pelos leigos com a timidez, porém ela é muito mais do que isto. É um transtorno mental grave que traz sofrimento e perdas de oportunidades para seu portador (D'El Rey, 2001; Lamberg, 1998). Ela é um transtorno ansioso de evolução crônica, além de ser reconhecida como passível de acarretar prejuízos graves em diferentes áreas da vida do indivíduo como trabalho, atividades sociais, escolaridade, relacionamento familiar, etc. (Schneier, Heckelman, Garfinkel, Del Bene \& Liebowitz, 1994).

Segundo Heimberg, Stein, Hiripi e Kessler (2000) e Stein, Walker e Forde (1996), recentes estudos epidemiológicos revelaram que a fobia social é mais prevalente do que se acreditava ser. Porém, o medo grave de falar em público constitui ainda um subtipo pouco reconhecido da fobia social em amostras comunitárias, assim como o impacto no funcionamento pessoal que este tipo de ansiedade social pode causar na vida do indivíduo. Estudos que utilizaram os critérios diagnósticos do DSM-III encontraram prevalências entre $2 \%$ e $3 \%$ para a fobia social (Pollard \& Henderson, 1988; Schneider, Johnson, Hornig, Liebowitz \& Weissman, 1992),

1 Endereço: Rua Bom Jesus, 274-B, São Paulo, SP, Brasil 03344-000. E-mail: g.delrey@bol.com.br enquanto que estudos que utilizaram os critérios diagnósticos do DSM-III-R e DSM-IV encontraram prevalências mais altas para a fobia social na população geral em um ano, ou seja, entre 7\% e 8\% (Kessler \& cols., 1994; Pélissolo, André, Moutard, Wittchen \& Lépine, 2000). Conforme Stein e cols. (1996), grande parte desta discrepância se deve às diferenças nos instrumentos diagnósticos utilizados em cada estudo. Por exemplo, a versão do Diagnostic Interview Schedule utilizada no Epidemiologic Catchment Area Study (Davidson, Hughes, George \& Blazer, 1993), avaliou três situações sociais (não incluindo falar em público), enquanto que a versão do Composite International Diagnostic Interview usada no National Comorbidity Survey (Kessler \& cols., 1994), avaliou seis situações sociais (incluindo falar em público). Os estudos que incluíram falar em público nas situações sociais avaliadas obtiveram uma maior prevalência. De acordo com Kessler, Stein e Berglund (1998), estudos que avaliaram a prevalência da fobia social ao longo da vida encontraram cerca de um terço das pessoas que preenchiam os critérios diagnósticos para o transtorno, relatando exclusivamente medo de falar em público (falar diante de um grande número de pessoas). D'El Rey e Almeida (2002) afirmam que em amostras de populações não-clínicas o medo de falar em público consiste na forma mais comum de ansiedade social, trazendo sofrimento e perdas de oportunidades. 
Uma questão ainda pouca estudada em estudos epidemiológicos é a proporção de pessoas na população geral com medo exclusivamente de falar em público e que são afetadas adversamente por ele, apresentando desta maneira conseqüências importantes para seu funcionamento pessoal e, desta forma poderiam receber o diagnóstico de fobia social. No estudo conduzido por Stein e cols. (1996), na cidade de Winnipeg no Canadá, com 499 pessoas da comunidade, 167 (34\%) pessoas reportaram ficar "muito mais nervosas do que as outras pessoas" quando falam para um grande grupo de pessoas (grande grupo não foi especificado neste estudo) e 114 pessoas (23\%) reportaram ficar "um pouco mais nervosas do que as outras pessoas" na mesma situação. No total, $10 \%$ das pessoas entrevistadas reportaram que o medo de falar em público resultou em grande interferência em suas atividades de trabalho (2\%), vida social (1\%), em sua educação/escolaridade (4\%), e/ou causou grande sofrimento (8\%). Cinco por cento relataram o medo de falar em público isoladamente, sem outros medos sociais adicionais.

No Brasil, não existem estudos que avaliem a prevalência do medo de falar em público (falar diante de um grande grupo de pessoas) e o impacto no funcionamento das pessoas acometidas por este tipo de ansiedade social. Até onde temos conhecimento, existem alguns estudos epidemiológicos que avaliaram a prevalência de transtornos mentais na população geral, entre eles as fobias, sem distinção entre elas (Almeida Filho \& cols., 1992; Andrade, Lólio, Gentil \& Laurenti, 1999). Em relação às características clínicas da fobia social existem diversos estudos teóricos, alguns são D'El Rey e Almeida (2002), Falcone (2001) e Barros Neto (2000). A grande maioria dos estudos diz respeito ao tratamento da fobia social, sendo a terapia cognitivo-comportamental e a terapia farmacológica as formas de tratamento mais estudadas (Picon \& cols., 1999; Rangé, 1994; Silveira \& Bicca, 2001). Acreditamos ser de alta relevância um estudo em nosso país relativo a este tema em específico, visto a carência de estudos deste tipo que verifique a prevalência do medo de falar em público, o impacto no funcionamento pessoal das pessoas acometidas, a procura de tratamento profissional e a relação deste tipo de medo com o diagnóstico de fobia social.

Este estudo teve como objetivo verificar a prevalência, o impacto no funcionamento pessoal e a procura de tratamento profissional para o medo grave de falar em público e sua relação com o diagnóstico de fobia social em uma amostra da população da cidade de São Paulo-SP.

\section{Método}

O estudo foi realizado com residentes de ambos os sexos e com idades entre 18 e 90 anos, com qualquer nível de escolaridade e estado civil da cidade de São Paulo-SP, Brasil. Os entrevistadores realizaram um treinamento de quatro horas para a aplicação randomizada de um questionário. Cada entrevistador ficou encarregado de entrevistar aproximadamente 110 pessoas aleatoriamente do bairro em que residia e trabalhava (vizinhos próximos). Os bairros estavam localizados respectivamente nas zonas leste, oeste, norte e sul da cidade de São Paulo.

Após uma breve introdução sobre o objetivo da pesquisa, assinatura do termo de consentimento e a coleta dos dados sócio-demográficos, o entrevistador perguntava ao entrevistado: "Quanto nervoso ou desconfortável ele ficaria se tivesse que falar diante de um pequeno grupo de pessoas (pequeno não foi especificado)?" e "Quanto nervoso ou desconfortável ele ficaria se tivesse que falar diante de um grande grupo de pessoas (ex: falar em público)?”. As respostas foram categorizadas em: "muito mais nervoso ou desconfortável do que as outras pessoas", "um pouco mais nervoso ou desconfortável do que as outras pessoas" e "não mais nervoso ou desconfortável do que as outras pessoas". Apenas para os entrevistados que responderam que ficariam "muito mais nervosos ou desconfortáveis do que as outras pessoas se tivessem que falar diante de um grande grupo de pessoas", era perguntado se o nervosismo ou o desconforto que ele sentiria viria de: "medo de fazer ou falar algo que lhe deixasse constrangido", "medo de que ocorresse um branco em sua mente na hora de falar", "medo de que não conseguisse continuar falando", "medo de dizer algo tolo ou sem sentido" e/ou "medo de gaguejar, tremer ou mostrar outros sinais de ansiedade". Para este item, as respostas variaram em: "sim", "não" e "não sei". Em seguida era perguntada a idade dos entrevistados quando eles tinham percebido pela primeira vez a dificuldade de falar diante de um grande público. Após, solicitava-se baseado em uma escala de 1 a 7 pontos $(1=$ de jeito nenhum; $4=$ de certa maneira; $6=$ bastante; 7 = totalmente) entregue ao entrevistado a responder quanto o medo de falar diante de um grande público o havia afetado em atividades/situações de trabalho (palestras e reuniões, encontrar um emprego, trocar de emprego, obter um emprego melhor, etc.); socialmente (relacionamento com a família, amigos, vizinhos, etc.); educação (apresentar trabalhos em sala de aula, chamada oral, etc.); e/ou se era algo que o incomodava pessoalmente. Para a análise deste item, somente as pessoas que responderam 6 ou 7 foram consideradas como "casos positivos". Esse critério bastante conservador foi utilizado para incluir apenas níveis graves de incapacitação e sofrimento ${ }^{2}$. E finalmente era perguntado ao entrevistado se o mesmo estava recebendo algum tipo de tratamento profissional para o medo de falar diante de um grande público. Em caso de resposta negativa era ainda perguntado o motivo de não estar em tratamento. Cada entrevista durou em média 16 minutos. Os participantes deste estudo, assinaram um termo de compromisso que explicava os objetivos da pesquisa e garantia seu anonimato (uma cópia do termo era entregue ao participante).

\section{Resultados}

\section{Características da amostra}

Foram entrevistadas 452 pessoas, sendo 212 (46,9\%) homens e $240(53,1 \%)$ mulheres. Cento e vinte e sete pessoas $(28,1 \%)$ residiam na zona leste da cidade, $109(24,1 \%)$ na zona oeste, $113(25,0 \%)$ na zona sul e 103 pessoas $(22,8 \%)$ residiam na zona norte. As idades das pessoas entrevistadas foram de 18 até 90 anos. As características sócio-demográficas da amostra da população pesquisada estão sumarizadas na Tabela 1.

2 Mesmo critério utilizado no estudo de Stein e cols. (1996). 
Tabela 1. Características sócio-demográficas $(N=452)$

\begin{tabular}{|c|c|}
\hline Características & $N(\%)$ \\
\hline \multicolumn{2}{|l|}{ SEXO } \\
\hline Masc. & $212(46,9)$ \\
\hline Fem. & $240(53,1)$ \\
\hline \multicolumn{2}{|l|}{ IDADE } \\
\hline $18-29$ & $104(23,0)$ \\
\hline $30-44$ & $183(40,5)$ \\
\hline $45-64$ & $98(21,7)$ \\
\hline$\geq 65$ & $67(14,8)$ \\
\hline \multicolumn{2}{|l|}{ ESTADO CIVIL } \\
\hline Solteiro & $131(29,0)$ \\
\hline Casado & $241(53,3)$ \\
\hline Separado/Divorciado & $44(9,7)$ \\
\hline Viúvo & $36(8,0)$ \\
\hline \multicolumn{2}{|l|}{ OCUPAÇÃO } \\
\hline Ativ. Remunerada & $269(59,5)$ \\
\hline Ativ. Não-remunerada & $101(22,4)$ \\
\hline Aposentado & $82(18,1)$ \\
\hline \multicolumn{2}{|l|}{ ESCOLARIDADE } \\
\hline Fund. Incompleto & $76(16,8)$ \\
\hline Fund. Completo & $102(22,6)$ \\
\hline Médio Incompleto & $92(20,4)$ \\
\hline Médio Completo & $85(18,8)$ \\
\hline Superior Incompleto & $46(10,2)$ \\
\hline Superior Completo & $51(11,2)$ \\
\hline \multicolumn{2}{|l|}{ RENDIMENTOS (S.M.) } \\
\hline 0 s.m. & $101(22,4)$ \\
\hline $1-5$ s.m. & $278(61,5)$ \\
\hline 6-10 s.m. & $53(11,7)$ \\
\hline$\geq 11$ s.m. & $20(4,4)$ \\
\hline \multicolumn{2}{|l|}{ ZONA RESIDENCIAL } \\
\hline Zona Leste & $127(28,1)$ \\
\hline Zona Oeste & $109(24,1)$ \\
\hline Zona Sul & $113(25,0)$ \\
\hline Zona Norte & $103(22,8)$ \\
\hline
\end{tabular}

\section{Prevalência do medo de falar em público}

Neste estudo, 143 pessoas (32\%) responderam que ficariam "muito mais nervosas ou desconfortáveis do que as outras pessoas" se tivessem que falar diante de um grande grupo de pessoas. Estas pessoas serão referidas como tendo "medo substancial de falar em público (MSFP)" ${ }^{3}$. Noventa e nove pessoas (22\%) responderam que ficariam "um pouco mais nervosas ou desconfortáveis do que as outras pessoas" nesta mesma situação (falar em público). Com relação à idade de início do medo de falar diante de um grande grupo, as pessoas com MSFP apresentaram como média 15 anos. Das pessoas com MSFP $(N=143)$, apenas $33(7 \%)$ relataram que ficariam "muito mais nervosas ou desconfortáveis do que as outras pessoas" se tivessem que falar diante de um pequeno grupo de pessoas concomitante ao medo de falar diante de um grande grupo.

3 Optamos por utilizar a denominação do estudo de Stein e cols. (1996).
Tabela 2. Pessoas com MSFP $(N=143)$ versus $\operatorname{sem} \operatorname{MSFP}(N=309)$

\begin{tabular}{|c|c|c|}
\hline \multirow{2}{*}{ Características } & n $(\%)$ & n $(\%)$ \\
\hline & MSFP & sem MSFP \\
\hline \multicolumn{3}{|l|}{ SEXO } \\
\hline Masc. & $42(29,4)$ & $170(55,0)$ \\
\hline Fem. & $101(70,6)$ & $139(45,0)$ \\
\hline \multicolumn{3}{|l|}{ IDADE } \\
\hline $18-29$ & $34(23,8)$ & $70(22,7)$ \\
\hline $30-44$ & $72(50,3)$ & $111(35,9)$ \\
\hline $45-64$ & $26(18,2)$ & $72(23,3)$ \\
\hline$\geq 65$ & $11(7,7)$ & $56(18,1)$ \\
\hline \multicolumn{3}{|l|}{ ESTADO CIVIL } \\
\hline Solteiro & $66(46,1)$ & $65(21,0)$ \\
\hline Casado & $49(34,3)$ & $192(62,2)$ \\
\hline Separado/Divorciado & $22(15,4)$ & $22(7,1)$ \\
\hline Viúvo & $6(4,2)$ & $30(9,7)$ \\
\hline \multicolumn{3}{|l|}{ OCUPAÇÃO } \\
\hline Ativ. Remunerada & $56(39,2)$ & $213(68,9)$ \\
\hline Ativ. Não-remunerada & $73(51,0)$ & $28(9,1)$ \\
\hline Aposentado & $14(9,8)$ & $68(22,0)$ \\
\hline \multicolumn{3}{|l|}{ ESCOLARIDADE } \\
\hline Fund. Incompleto & $35(24,5)$ & $41(13,3)$ \\
\hline Fund. Completo & $43(30,1)$ & $59(19,1)$ \\
\hline Médio Incompleto & $28(19,5)$ & $64(20,7)$ \\
\hline Médio Completo & $19(13,3)$ & $66(21,4)$ \\
\hline Superior Incompleto & $9(6,3)$ & $37(11,9)$ \\
\hline Superior Completo & $9(6,3)$ & $42(13,6)$ \\
\hline \multicolumn{3}{|l|}{ RENDIMENTOS (S.M.) } \\
\hline 0 s.m. & $73(51,0)$ & $28(9,1)$ \\
\hline $1-5$ s.m. & $62(43,4)$ & $216(69,8)$ \\
\hline 6-10 s.m. & $8(5,6)$ & $45(14,6)$ \\
\hline$\geq 11$ s.m. & $0(0,0)$ & $20(6,5)$ \\
\hline \multicolumn{3}{|l|}{ ZONA RESIDENCIAL } \\
\hline Zona Leste & $31(21,7)$ & $96(31,1)$ \\
\hline Zona Oeste & $36(25,2)$ & $73(23,6)$ \\
\hline Zona Sul & $51(35,6)$ & $62(20,1)$ \\
\hline Zona Norte & $25(17,5)$ & $78(25,2)$ \\
\hline
\end{tabular}

\section{Características sócio-demográficas associadas ao medo de falar em público}

Neste estudo, a maioria das pessoas com $\operatorname{MSFP}(N=143)$ era do sexo feminino $(70,6 \%)$, com idades entre 30 e 44 anos $(50,3 \%)$, solteiras $(46,1 \%)$, sem atividade remunerada $(51,0 \%)$ no momento da entrevista, com ensino fundamental completo $(30,1 \%)$, com rendimentos iguais a 0 (zero) salários mínimos $(51,0 \%)$ por mês e residentes na zona sul $(35,6 \%)$ da cidade de São Paulo. A Tabela 2 apresentara o contraste sócio-demográfico das pessoas com MSFP versus o das pessoas sem MSFP.

\section{Cognições ansiosas associadas ao medo de falar em público}

As seguintes cognições foram relatadas pelas pessoas com MSFP $(N=143)$ : medo de fazer ou falar algo que cause constrangimento (64\%), medo de que dê um "branco na mente" na hora de falar (82\%), medo de não conseguir 
continuar falando $(50 \%)$, medo de dizer algo tolo ou sem sentido (43\%) e medo de gaguejar, tremer ou mostrar outros sinais de ansiedade (94\%). As pessoas com MSFP relataram uma ou mais destas cognições anteriores, sendo estas consideradas comuns em pessoas portadoras de fobia social com respeito a falar em público.

\section{Impacto no funcionamento pessoal do medo de falar em público}

Neste estudo, 143 pessoas reportaram "medo substancial de falar em público (MSFP)", porém quantas destas pessoas foram realmente afetadas adversamente pelo medo de falar em público? Mais precisamente, quantas pessoas com MSFP preenchiam os critérios diagnósticos para fobia social, segundo o DSM-IV-TR. Em nosso estudo, interferência nas rotinas e sofrimento foram medidos através da escala de 1 a 7 pontos $(1=$ de jeito nenhum; $4=$ de certa maneira; $6=$ bastante; $7=$ totalmente). Como relatado anteriormente, para incluir apenas níveis graves de interferência e sofrimento, somente as pessoas que responderam 6 ou 7 neste item foram consideradas como "casos positivos".

Sessenta e uma pessoas (43\% dos indivíduos com MSFP e $13 \%$ dos entrevistados) foram consideradas como "casos positivos", respondendo desta maneira que o medo de falar em público afetou adversamente uma ou mais importantes áreas de suas vidas e/ou causou sofrimento acentuado. Assim, 41 pessoas (29\% das pessoas com MSFP e 9\% dos entrevistados) relataram que o medo de falar diante de um grande público as afetou significativamente em situações de trabalho, tais como palestras, reuniões, encontrar um emprego, trocar de emprego, etc.; 19 (13\% das pessoas com MSFP e 4\% dos entrevistados) relataram marcante interferência em suas atividades sociais, tais como relacionamento com a família, fazer amizades, etc.; 54 (38\% das pessoas com MSFP e 12\% dos entrevistados) reportaram grande interferência em suas atividades escolares, tais como apresentar trabalhos em sala de aula, chamada oral, etc.; e 61 pessoas (42\% das pessoas com MSFP e $13 \%$ dos entrevistados), ou seja, todas, relataram sofrimento acentuado por terem este tipo de medo. $\mathrm{O}$ Gráfico 1 mostra a proporção das pessoas com MSFP em relação ao impacto no funcionamento (trabalho, vida social e educação) e sofrimento, considerando-se a escala de 1 a 7 pontos.

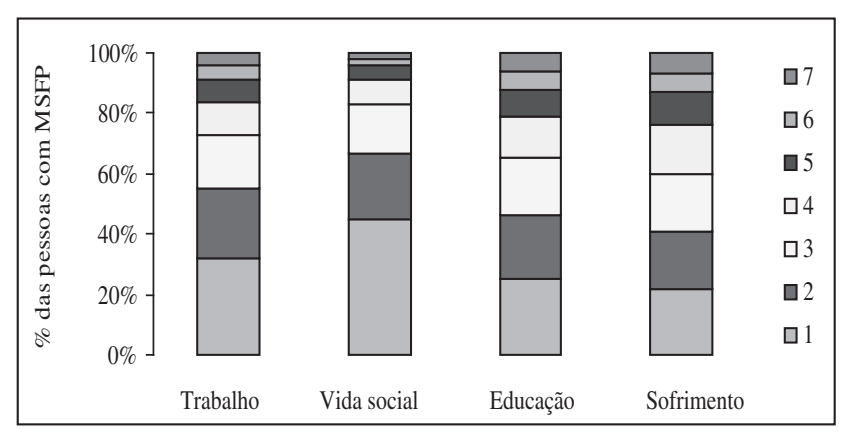

Gráfico 1. Porcentagem das pessoas com MSFP em relação ao impacto no funcionamento e sofrimento (escala de 1 a 7 pontos).

\section{Medo de falar diante de um grande público isoladamente $^{4}$}

Quantas destas pessoas consideradas neste estudo como "casos positivos $(N=61)$ " apresentaram o medo de falar diante de um grande público isoladamente, em oposição às pessoas que sofrem da forma generalizada da fobia social, na qual o medo de falar em público é apenas uma das manifestações deste subtipo do transtorno? Consideramos as pessoas incluídas nos "casos positivos" que apresentavam apenas o medo de falar diante de um grande grupo de pessoas. Ou seja, as pessoas que além de reportarem o medo de falar diante de um grande público reportaram também o medo de falar diante de um pequeno grupo de pessoas foram excluídas desta análise. Trinta pessoas (49\% dos "casos positivos" e 7\% dos entrevistados) apresentaram o medo de falar em público isoladamente, sem evidências de outros tipos de medos sociais.

\section{Procura de tratamento para o medo de falar em público}

Visto que as pessoas dentro do grupo de "casos positivos" poderiam receber o diagnóstico de fobia social. Quantas destas pessoas estão recebendo tratamento profissional para este medo? No grupo de "casos positivos" $(N=61)$, apenas sete pessoas $(11 \%)$ estavam recebendo tratamento profissional para o medo de falar em público e 54 (89\%) não estavam recebendo nenhum tipo de tratamento. Duas pessoas (3\%) estavam recebendo tratamento psicológico para o medo e cinco $(8 \%)$ estavam em tratamento combinado, ou seja, psicológico e farmacológico.

\section{Discussão}

Nós conduzimos este estudo, para verificar a prevalência, o impacto no funcionamento pessoal e a procura de tratamento profissional para o medo de falar em público em uma amostra da população da cidade de São Paulo-SP, visto que até onde sabemos não existem estudos epidemiológicos relacionados a este tema no Brasil. Encontramos 32\% da população entrevistada com medo substancial de falar em público (MSFP) e 22\% relatando "que ficariam um pouco mais nervosas do que as outras pessoas se tivessem que falar em público". Estes achados são semelhantes aos encontrados na pesquisa de Stein e cols. (1996), ou seja, 34\% e 23\%, respectivamente.

Treze por cento das pessoas com MSFP tinham o medo de falar em público associado com significativa interferência em uma ou mais das seguintes áreas, como trabalho, vida social e escolaridade e/ou sofrimento acentuado e apresentavam ainda cognições típicas daquelas observadas em pacientes com fobia social, conforme descrito por Anderson (1997), D’El Rey (2001), Falcone (2001), Heimberg, Hope, Dodge e Becker (1990) e Taylor, Woody, Koch, McLean. A idade de início do medo de falar em público se deu em média aos 15 anos, sendo este um dado semelhante ao descrito por

4 Em nosso estudo, "grande público" e "pequeno grupo de pessoas" não foram explicitados para os entrevistados durante as entrevistas. 
Magee, Eaton, Wittchen, McGonagle e Kessler (1996) e Öst (1987) para início da fobia social, ou seja, aproximadamente aos 16 anos. Por estas razões descritas anteriormente, parece razoável concluir que o medo grave de falar em público (ex: aquele associado a grande interferência nas rotinas e sofrimento acentuado) pertence ao espectro diagnóstico da fobia social, como também afirmam Kessler e cols. (1998) e Stein e cols. (1996).

Nós confirmamos os achados prévios de que existe uma taxa elevada de pessoas com medo grave de falar em público entre a população geral (Heimberg \& cols., 1990; Kessler \& cols., 1998; Stein \& cols., 1996). Ao interpretar este achado, deve ser mantido em mente que nós perguntamos às pessoas: Quanto nervoso ou desconfortável você ficaria (...)? e não: Quanto nervoso ou desconfortável você fica (...)? Conseqüentemente, é provável que entre os entrevistados estão algumas pessoas que hipotetizaram que ficariam extremamente nervosas e desconfortáveis se tivessem que falar em público, mesmo não podendo realmente ter tido a oportunidade de testar esta hipótese.

As mulheres foram aproximadamente duas vezes mais afetadas por este tipo de medo do que os homens, semelhante ao descrito por Stein e cols. (1996) e Costello (1982) em seus estudos.

Quando comparamos os entrevistados com medo substancial de falar em público (MSFP) com os entrevistados sem medo substancial de falar em público (sem MSFP), encontramos para os indivíduos com MSFP mais pessoas solteiras, sem atividades remuneradas e com recebimentos mensais de 0 (zero) salários mínimos.

Em contrapartida, encontramos para as pessoas sem MSFP mais indivíduos casados, com atividades remuneradas e com recebimentos mensais entre um a cinco salários mínimos. As pessoas com MSFP tinham uma menor escolaridade do que as pessoas sem MSFP, ou seja, ensino fundamental nas pessoas com MSFP e ensino médio completo nas pessoas sem MSFP, estes achados são semelhantes aos descritos por Heimberg e cols. (1990) nas pessoas com diagnóstico de fobia social.

O medo de falar em público em nossa pesquisa interferiu significativamente mais na área da escolaridade/educação $(12 \%)$, do que em outras áreas avaliadas (trabalho $=9 \% \mathrm{e}$ vida social $=4 \%$ ). Talvez este achado possa explicar a baixa escolaridade encontrada nas pessoas com MSFP em comparação com as pessoas sem MSFP. Este achado é compatível com os dados relatados por Bruch, Fallon e Heimberg (2003), relativos à escolaridade das pessoas com fobia social.

Em nosso estudo, aparentemente $7 \%$ dos entrevistados apresentaram o medo de falar em público isoladamente. Isto sugere que o medo de falar em público pode aparecer como única manifestação do quadro fóbico social, e não apenas como mais uma das manifestações da forma generalizada da fobia social, como descrevem Kessler e cols. (1998). Esta forma de fobia social relacionada somente com o falar em público, corresponde na DSM-IV-TR à fobia social circunscrita ou nãogeneralizada, como afirmam Stein e Chavira (1998).

O DSM-IV-TR (APA, 2002) explicita em seus critérios diagnósticos para fobia social que o medo deve interferir significativamente nas rotinas ocupacionais, sociais ou acadêmicas e/ou causar sofrimento acentuado à pessoa por ser portadora da fobia. As pessoas consideradas como "casos positivos" $(N=61)$ que reportaram que o medo de falar em público afetou adversamente uma ou mais importantes áreas de sua vidas e/ou causou sofrimento acentuado, poderiam receber o diagnóstico de fobia social segundo o DSM-IV-TR (sem considerarmos o critério G de exclusão de um transtorno físico ou efeitos fisiológicos diretos de uma substância psicoativa ou outro transtorno mental explicando melhor o quadro fóbico). Cabe lembrar que todas as pessoas consideradas "casos positivos $(N=$ $61)$ " reportaram sofrimento acentuado por ter o medo grave de falar em público.

Com relação à procura de tratamento profissional para o medo grave de falar em público associado à interferência e sofrimento, podemos hipotetizar que o baixo número de pessoas do grupo de "casos positivos" em tratamento para este problema, provavelmente se deve ao fato das pessoas leigas interpretarem este tipo de medo muito mais em termos de timidez do que se atribuir estes sintomas a um transtorno mental ou problema clínico que mereça atenção profissional, como afirmam D'El Rey (2001), Lamberg (1998), Magee e cols. (1996) e Thompson, Burns, Bartko, Boyd e Bourdon (1988).

Por interferir nas rotinas de trabalho, vida social e escolaridade e causar sofrimento, o medo de falar em público deve ser encarado como um problema sério de saúde mental. Programas preventivos deveriam ser criados em ambulatórios, clínicas, escolas e empresas para se identificar, aconselhar e tratar quando necessário às pessoas com este tipo de medo.

\section{Conclusão}

Visto que existe uma carência no Brasil de estudos de prevalência em relação ao medo grave de falar em público, o impacto no funcionamento pessoal das pessoas acometidas e a relação deste tipo de medo com a fobia social, acreditamos que este estudo venha a dar início a novas pesquisas sobre o tema, suprindo desta maneira esta lacuna na literatura psicológica brasileira.

Neste estudo, $32 \%$ das pessoas entrevistadas apresentaram medo excessivo de falar diante de um grande público. Além disso, $13 \%$ dos entrevistados relataram que o medo de falar em público resultou em grande interferência em suas vidas e/ou causou sofrimento acentuado. Apenas $11 \%$ das pessoas com medo excessivo de falar em público associado a cognições fóbicas e interferência e sofrimento estavam recebendo tratamento profissional para o medo.

Este estudo apóia a inclusão de formas graves do medo de falar em público, tais como aquelas que interferem no trabalho, na vida social, na escolaridade ou cause sofrimento acentuado no constructo diagnóstico da fobia social, para que as pessoas acometidas por essa forma de ansiedade social possam receber a atenção necessária e conseqüentemente o tratamento adequado.

Sugerimos ainda, que em um próximo estudo seja verificado com as pessoas portadoras da forma grave do medo de falar em público e que não estejam em tratamento, qual o motivo que leva estas pessoas a não estarem recebendo tratamento profissional. 


\section{Referências}

Almeida Filho, N., Mari, J. J., Coutinho, E., França, J. F., Fernandes, J. G., Andreoli, S. B. \& Busnello, E. D. (1992). Estudo multicêntrico de morbidade psiquiátrica em áreas urbanas brasileiras (Brasília, São Paulo, Porto Alegre). Revista ABP-APAL, 14(3), 93-104.

Andrade, L. H. S. G., Lólio, C. A., Gentil, V. \& Laurenti, R. (1999). Epidemiologia dos transtornos mentais em uma área definida de captação da cidade de São Paulo, Brasil. Revista de Psiquiatria Clínica, 26(5), 257-261.

APA. (2002). DSM-IV-TR: Manual diagnóstico e estatístico de transtornos mentais ( $4^{\mathrm{a}}$ ed.). Porto Alegre: Artmed.

Barros Neto, T. P. (2000). Fobia social: Perfil clínico, comorbidade e diagnóstico diferencial. Revista de Psiquiatria Clínica, 27(6), 309-315.

Bruch, M. A., Fallon, M. \& Heimberg, R. G. (2003). Social phobia and difficulties in ocupacional adjustament. Journal of Conseling Psychology, 50(1), 109-117.

Costello, C. G. (1982). Fears and phobias in women: A community study. Journal of Abnormal Psychology, 91(3), 280-286.

Davidson, J. T., Hughes, D. L., George, L. K. \& Blazer, D. G. (1993). The epidemiology of social phobia: Findings from the epidemiological catchment area study. Psychological Medicine, 23(4), 709-718.

D’El Rey, G. J. F. (2001). Fobia social: Mais do que uma simples timidez. Arquivos de Ciências da Saúde da Unipar, 5(3), 273276.

D’El Rey, G. J. F. \& Almeida, I. P. (2002). Transtornos fóbicos na atualidade. Integração: Ensino, Pesquisa e Extensão, 8(28), 25-29.

Falcone, E. O. (2001). O processamento cognitivo da ansiedade na fobia social. Revista de Psiquiatria Clínica, 28(6), 309-312.

Heimberg, R. G., Hope, D. A., Dodge, C. S. \& Becker, R. E. (1990). DSM-III-R subtypes of social phobia: Comparison of generalized social phobics and public-speaking phobics. Journal of Nervous and Mental Disease, 178(1), 172-179.

Heimberg, R. G., Stein, M. B., Hiripi, E. \& Kessler, R. C. (2000). Trends in the prevalence of social phobia in the United States: A synthetic cohort analysis of changes over four decades. European Psychology, 15(1), 29-37.

IBGE. (2003). Resultados do censo de população - 2000. São Paulo: IBGE.

Kessler, R. C., McGonagle, K. A., Zhao, S., Nelson, C. B., Hughes, M., Eshleman, S., Wittchen, H.-U. \& Kendler, K. S. (1994). Lifetime and 12-month prevalence of DSM-III-R psychiatric disorders in the United States: Results from the national comorbidity survey. Archives of General Psychiatry, 51(1), 8-19.

Kessler, R. C., Stein, M. B. \& Berglund, P. (1998). Social phobia subtypes in the national comorbidity survey. American Journal of Psychiatry, 155(5), 613-619.

Lamberg, L. (1998). Social phobia: Not just another name for shyness. Journal of American Medical Academy, 280(8), 685-686.
Magee, W. J., Eaton, W., Wittchen, H.-U., McGonagle, K. A. \& Kessler, R. C. (1996). Agoraphobia, simple phobia, and social phobia in the national comorbidity survey. Archives of General Psychiatry, 53(2), 159-168.

Öst, L. G. (1987). Age of onset of different phobias. Journal of Abnormal Psychology, 96(3), 223-229.

Pélissolo, A., André, C., Moutard, F., Wittchen, H.-U. \& Lépine, J. P. (2000). Social phobia in the community: Relationship between diagnostic threshold and prevalence. European Psychology, 15(1), 25-28.

Picon, P., Kapczinski, F., Fichbein, B., Ballester, D., Guauer, G. C., Pereira, M. O., Silveira, R. O. \& Fitjmann, R. P. (1999). Terapia cognitivo-comportamental e farmacológica da fobia social: Uma revisão. Revista de Psiquiatria do Rio Grande do Sul, 21(1), 52-65.

Pollard, C. A. \& Herderson, J. G. (1988). Four types of social phobia in a community sample. Journal of Nervous and Mental Disease, 176(3), 440-445.

Rangé, B. P. (1994). Terapia cognitivo-comportamental da fobia social. Jornal Brasileiro de Psiquiatria, 43(6), 327-331.

Schneier, F. R., Heckelman, L. R., Garfinkel, R., Del Bene, D. \& Liebowitz, M. R. (1994). Functional impairment in social phobia. Journal of Clinical Psychiatry, 55(3), 322-331.

Schneider, F. R., Johnson, J., Hornig, C. D., Liebowitz, M. R. \& Weissman, M. M. (1992). Social phobia: Comorbidity and morbidity in a epidemiologic sample. Archives of General Psychiatry, 49(2), 282-288.

Silveira, R. O. \& Bicca, C. (2001). Terapia cognitiva no tratamento da fobia social. Revista Brasileira de Psicoterapia, 3(1), 7990.

Stein, M. B. \& Chavira, D. A. (1998). Subtypes of social phobia and comorbidity with depression and other anxiety disorders. Journal of Affective Disorders, 50(1), 11-16.

Stein, M. B., Walker, J. R. \& Forde, D. R. (1996). Public-speaking fears in a community sample: prevalence, impact on functioning, and diagnostic classification. Archives of General Psychiatry, 53(2), 169-174.

Taylor, S., Woody, S., Koch, W. J., McLean, P. \& Anderson, K. W. (1997). Cognitive restructuring in the treatment of social phobia: Efficacy and mode of action. Behaviour Modification, 21(4), 487-511.

Thompson, J. W., Burns, B. J., Bartko, J., Boyd, J. H. \& Bourdon, K. H. (1988). The use of ambulatory service by persons with and without phobia. Medical Care, 26(2), 183-198.

Recebido em 14.05.2004

Primeira decisão editorial em 31.01.2005

Versão final em 23.02.2005

Aceito em 26.07.2005 